\title{
Der Botanische Garten „Julia \& Alexander N. Diomides“ in Athen
}

\author{
Hilke Steinecke
}

\section{Abstract}

The history and some plants of the botanical garden of Athens are described.

Zusammenfassung

Die Geschichte des botanischen Gartens und einige Pflanzen werden beschrieben.

\section{Kurzer Überblick über die Geschichte}

Der zur Universität von Athen gehörende botanische Garten befindet sich $14 \mathrm{~km}$ westlich des Stadtzentrums von Athen, etwa $3 \mathrm{~km}$ von der Küste entfernt. Der Garten wird zwar von einer Buslinie angesteuert, doch empfiehlt es sich für Ortsunkundige sehr, ein Taxi zu nehmen. Bei der Planung eines Besuches dieses Gartens sollte eine Verwechslung mit einem anderen, allerdings kaum einen Hektar großen Garten vermieden werden, denn dieser kleine, öffentlich nicht zugängliche und in der Innenstadt Athens gelegene Garten wird in manchen touristischen Stadtführern als der botanische Garten der Universität beschrieben.

Der hier vorgestellte Garten wurde 1952 gegründet und ist seit 1975 öffentlich zugänglich. Benannt wurde er nach dem Athener AlexanDER DiOMIdes (1875-1950) und seiner Ehefrau. Diomides gilt als eine der führenden griechischen Persönlichkeiten des 20. Jh. Der Rechts- und Wirtschaftswissenschaftler promovierte 1898 in Berlin und erhielt bereits im Alter von nur 30 Jahren eine Professur an der Athener Universität. Zudem schrieb er für verschiedene Athener und internationale Zeitschriften, arbeitete bis 1949 in der Führungsetage der Nationalbank von Griechenland und war ein aktiver Politiker in verschiedenen Ämtern bis hin zum Premierminister. Mit der Einrichtung eines botanischen Gartens, der allerdings erst im Jahr 1952, also nach seinem Tod, offiziell anerkannt wurde, erfüllte sich Diomides einen großen Wunsch. Ein großes, naturbelassenes Gelände wurde hierfür vom Agrarministerium zur Verfügung gestellt. Die Arbeiten zur Einrichtung des Gartens zogen sich über einen recht langen Zeitraum hin und erst nach ihrem Abschluss im Jahr 1975 konnte der Garten für die Öffentlichkeit freigegeben werden. Nach Diomides' testamentarischem Willen soll der Garten der Allgemeinheit dienen, unabhängig und nicht profitorientiert sein. Er wird finanziell von Diomides' Vermögen getragen. Vom Gründer wurde festgelegt, dass der Garten von einem fünfköpfigen Gremium verwaltet wird, dem auch der Rektor der Athener Universität, der Leiter des botanischen Instituts der Universität und der Präsident der griechischen Nationalbank angehören. Die derzeitige wissenschaftliche Leiterin des Gartens ist die Botanikerin IRINI VALLIANATOU.

\section{Der Garten heute}

Der Garten umfasst ein großes Areal von 186 ha. Der größte Anteil ist naturbelassen, während nur 20 ha kultiviert werden. Für dieses große Gelände sind insgesamt 15 Mitarbeiterinnen und Mitarbeiter zuständig, die neben den gärtnerischen Pflegearbeiten vielfältige Aufgaben zu erledigen haben. Im Sommer besteht große Brandgefahr, zumal eine Straße das Gelände durchzieht und die Gefahr besteht, dass unachtsam aus dem Auto geworfene Zigarettenkippen Feuer entstehen lassen. Zwei Mitarbeiter halten deshalb im Sommer Feuerwache, außerdem muss zur Minderung der Brandgefahr das trockene Gras geschnitten werden. Der Garten besitzt ein eigenes Feuerlöschauto.

Außer der Systematikerin Irini Vallianatou arbeitet im Garten noch ein zweiter Wissenschaftler, der sich u. a. besonders mit der Samenund Gewebekultur von Pflanzen beschäftigt. Neben den Büroräumen der Gartenverwaltung verfügt der Garten über ein Labor. Dort sind das Herbarium, das über 10000 Belege umfasst, sowie die Samenbank untergebracht. Der Garten ist in das Projekt „NATURA 2000“ zum 
Schutz und zur Erforschung der griechischen Flora involviert und der Pflanzenbestand des Gartens wird wissenschaftlich dokumentiert sowie in einer Datenbank festgehalten.

\section{Das naturbelassene Gelände}

Das nicht- oder nur extensiv bewirtschaftete Gelände des Gartens zieht sich bis auf einen Felsrücken, von dem man einen schönen Blick über den ganzen Garten hat. Hier findet man überwiegend Phrygana-Vegetation mit stacheligen Sträuchern wie Sarcopoterium spinosum und Euphorbia acanthothamnos. Im unteren Bereich gedeiht ein lichter Wald aus Pinus halepensis. Es handelt sich um einen der ältesten Wälder Attikas. Die Kiefern hier sind etwa 80 Jahre alt und streng geschützt. Kein Baum darf gefällt werden, was manchmal im Konflikt mit gestalterischen Maßnahmen des Gartens steht, da im Unterwuchs der Kiefern kaum andere Pflanzen gedeihen. Zwischen die Aleppo-Kiefern wurden einige andere Gehölze gepflanzt, die in Attika nicht wild vorkommen, darunter auch Pinus brutia und Cupressus sempervirens.

Geht man nur ein paar Schritte vom Weg ab, kann die Vielfalt der einige hundert im Gelände auftretenden Wildarten erahnt werden. Im April gehören Cistus incanus und Phlomis fruticosa zu den auffälligsten Gewächsen. Einige Lippenblütler wie z. B. Stachys cretica stehen dann in Blüte und die ersten Orchideen erscheinen, während Cyclamen graecum seine Kapseln an gedrehten Fruchtstielen der Erde zuwendet.

Die Natur hat hier im Ballungsraum Athen ein sicheres Rückzugsgebiet gefunden. Zusammen mit den Pflanzen leben auch viele Tiere im Garten. Mit ein wenig Glück kann man beispielsweise Raupen des Schwalbenschwanzes oder sogar griechische Landschildkröten entdecken.

Abb. 1 (oben): Einblick in die Samenbank im Botanischen Garten Athen.

Abb. 2 (Mitte): Im naturbelassenen Gelände des Gartens.

Abb. 3 (unten): Der Garten ist Lebensraum für viele heimische Tier- und Pflanzenarten; hier eine Raupe des Schwalbenschwanzes an Weinraute.
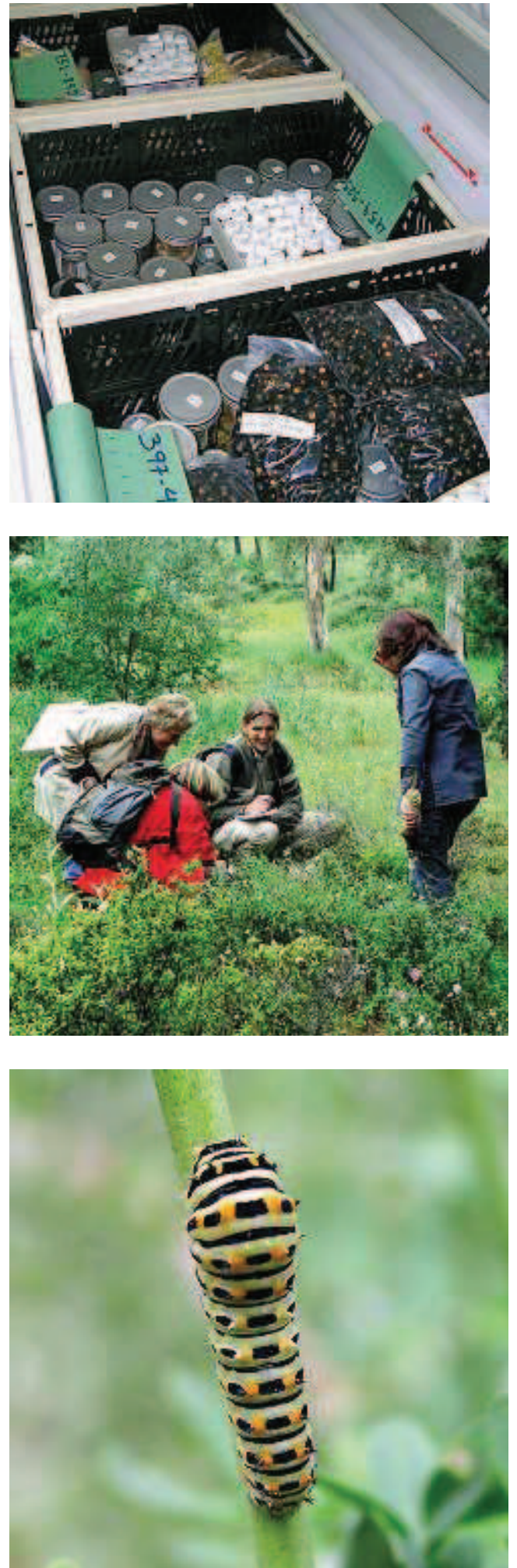

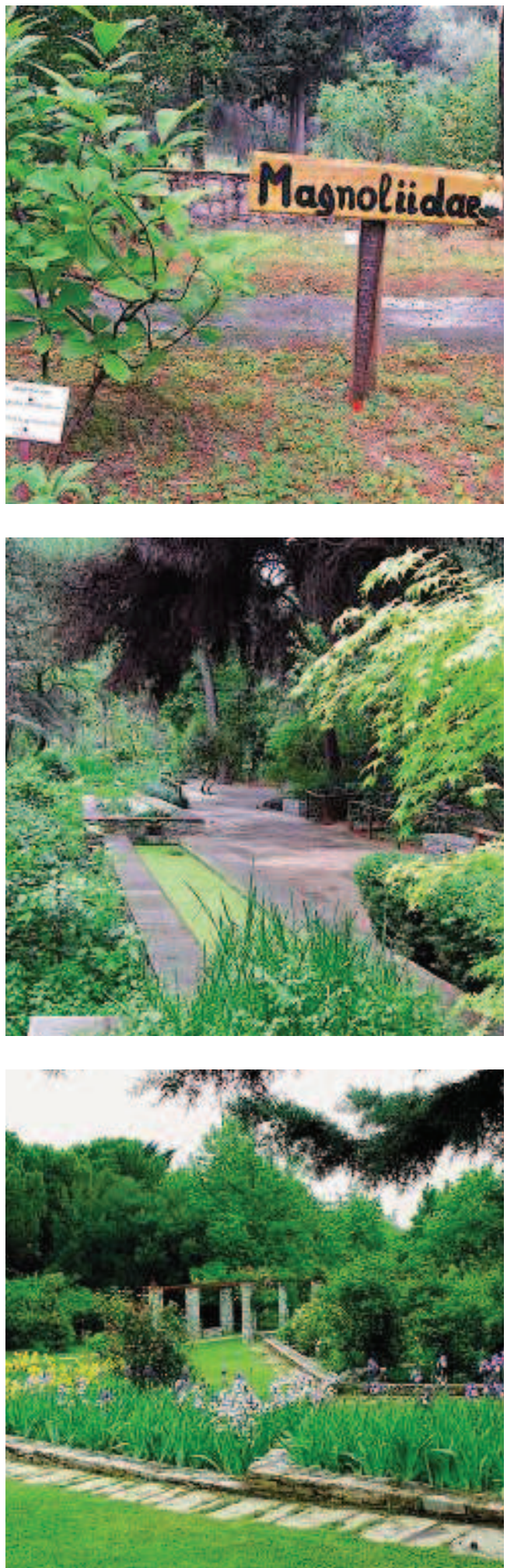

4. Der kultivierte Bereich des Gartens Hier werden in einem Arboretum verschiedene Bäume aus aller Welt gezeigt. Zudem gibt es einen größeren Bereich mit Beeten, auf denen diverse Zierpflanzen kultiviert werden, einen Themengarten mit historischen Pflanzen, die vor allem in der griechischen Antike von Bedeutung waren sowie einen Bereich mit aromatischen und für die Medizin wichtigen Arten. Ein weiterer Teil des Gartens ist wirtschaftlich bedeutenden Nutzpflanzen gewidmet. In einem kleinen Foliengewächshaus werden Arten gehalten, die gegenüber Feuchtigkeit oder niedrigen Temperaturen empfindlich sind, darunter z. B. Kakteen, verschiedene Euphorbien, Kaffee und Zuckerrohr. Außerdem ist im Garten ein kleines System angelegt. Alle thematischen Bereiche des Gartens sowie einzelne Pflanzen sind durch liebevoll gestaltete Holzschilder, die von Irini Vallianatou mit der Hand beschrieben werden, ausgezeichnet. Insgesamt werden in diesem Gartenbereich 3000 Arten kultiviert

\section{Ein Rundgang durch den Garten}

Vom Verwaltungsgebäude ausgehend gelangt man zunächst durch das kleine System, in dem verschiedenen Pflanzengruppen wie Moosen, Farnen, Nacktsamern sowie einigen ausgewählten ein- und zweikeimblättrigen Pflanzen Beete gewidmet sind. Von dort passiert der Besucher die Medizinalpflanzenabteilung. Hier werden klassische Heilpflanzen wie Fingerhut, Johanniskraut, Lavendel, Rizinus, Alraune und Beifuß gezeigt. Im Bereich der Nutzpflanzen wachsen Korkeiche, Tabak, Wein, Ölbaum und Zitrusgewächse. Ein gepflasterter Pfad führt entlang mehrerer rechteckiger Wasserbecken, über die man auch auf Trittsteinen laufen kann. Wo im Frühling das Wasser mit einer grünen Schicht aus Wasserlinsen und Azolla überzogen ist, stehen im Sommer prächtig die Lotosblumen ( $\mathrm{Ne}$ -

Abb. 4 (oben): Beispiel für handgeschriebene Schilder in der systematischen Abteilung.

Abb. 5 (Mitte): Wasserbecken mit Wasserlinsen.

Abb. 6 (unten): Im bunt blühenden Staudenbereich. 
lumbo nucifera und $N$. lutea) und blühen viele Seerosen.

Von dort führt der Weg in den mittleren, großzügig gestalteten Bereich mit Zierpflanzen. Hier gibt es 15 rechteckige, mit Natursteinmauern eingefasste Beete. Über 20 Wasserbecken sowie Rasenflächen prägen das Bild. Dazwischen wurden kleinere Hecken und Gehölzgruppen gepflanzt. Auffällige Bäume sind hier Magnolia grandiflora, Retama monosperma, Ginkgo biloba und Liquidambar orientalis. Eine wahre Pracht ist im April der Styraxbaum (Styrax officinalis), der wie eine Wolke von weißen Blüten überzogen ist.

An mehreren ebenfalls aus Natursteinen errichteten Pergolen ranken verschiedene Kletterpflanzen, im Frühling blühen hier Periploca graeca, Wisteria sinensis und verschiedene Rosen (z.B. R. banksiae). Etwas später blühen dann Geißblatt, Passionsblume und Jasmin. Große Kanarische Dattelpalmen im Hintergrund bieten diesem Gartenbereich einen besonders schönen Aspekt. Im Frühling fallen unter den Beetpflanzen vor allem verschiedene Iris, Rosen, Asiatischer Hahnenfuß und Pfingstrosen auf. Erst im Sommer entfalten Indisches Blumenrohr und Bananenstauden ihre volle Schönheit.

Gleich nebenan befindet sich ein Beet mit historischen Pflanzen. Hier wachsen beispielsweise Acanthus, dessen Blätter Vorbilder für die Gestaltung von Säulenkapitellen waren, und Gefleckter Schierling, aus dem der tödlich giftige Schierlingstrunk hergestellt wurde. Laut griechischer Mythologie brachte Prometheus das göttliche Feuer in einem hohlen Stängel von Ferula communiszu den Menschen. Lorbeer der heiligen Haine sollte in dieser Thematik nicht fehlen.

Den größten Teil des gestalteten Gartengeländes nimmt das Arboretum ein, in dem Gehölze aus aller Welt gepflanzt wurden. Es gibt

Abb. 7 (oben): Blauregen an einer Pergola vor einem terrassierten Beet.

Abb. 8 (Mitte): Die Gartenleiterin (Mitte) führt uns durch den Garten.

Abb. 9 (unten): Styrax officinalis.


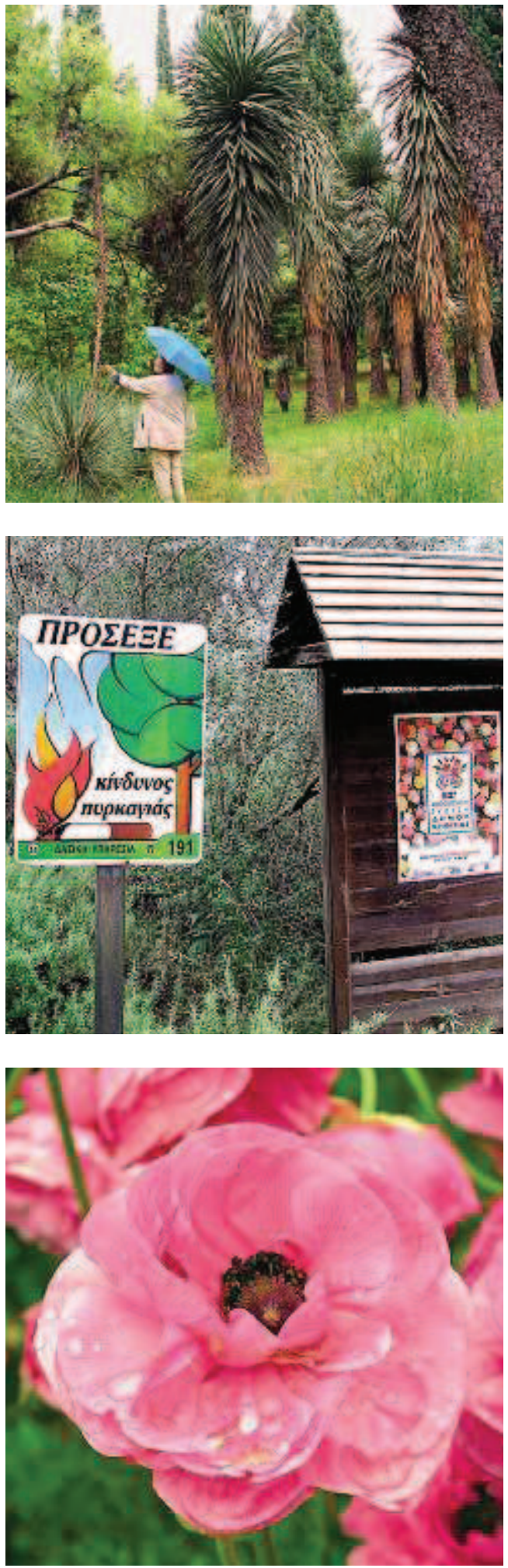

Bereiche mit Pflanzen aus Ozeanien, Europa und dem Mittelmeergebiet, Asien, Südafrika, Nord- und Zentralamerika sowie Südamerika. In der australischen Abteilung beispielsweise beeindrucken große Eukalypten, Akazien, Melaleuca und Callistemon. In "Nordamerika“ gedeihen prächtige und mehrere Meter hohe Exemplare von Yucca baccata sowie Dasylirion, Nolina und mehrere sehr große Exemplare von Agave americana. Der europäische Bereich beherbergt u. a. die beiden in Europa heimischen Palmen Phoenix theophrastii und Chamaerops humilis sowie Eichen, Ahorn, Eschen und Cedrus atlantica.

\section{Ein Garten für die Öffentlichkeit}

Der Garten ist wochentags von 8-14 h und an Wochenenden von 10-15 h geöffnet. Zahlreiche Schulklassen aller Altersstufen, aber auch Studenten, die die heimische Flora kennen lernen möchten, besuchen den Garten. Seit einiger Zeit wurde die Durchführung von Führungen an einen privaten Anbieter abgegeben. Für Kindergruppen, die nach einem Rundgang noch etwas Entspannung suchen, wurde kürzlich ein Spielplatz eingerichtet. Diejenigen, die sich etwas ausführlicher über den Garten informieren wollen, können eine großzügig illustrierte Gartenbroschüre bekommen.

\section{Dank}

Ganz herzlicher Dank gilt Irini Vallianatou, die sich so viel Zeit dafür genommen hat, uns mit großer Begeisterung die Schönheiten ihres Gartens zu zeigen.

\author{
Literatur \\ National and capodistrian University of Athens (Hrsg.) \\ o. J.: Julia \& Alexander N. Diomides Botanic Garden. \\ - Athen.
}

Abb. 10 (oben): Im Arboretum, Abteilung Amerika, mit riesigen Yucca-Exemplaren.

Abb. 11 (Mitte): Warnung vor Waldbrandgefahr im botanischen Garten.

Abb. 12 (unten): Ranunculus asiaticus wirkt aus der Ferne wie eine Rose. 